\title{
Vision and Force Feedback Control for Microassembly Process
}

\author{
Chen Zhi yao, Ye Xin, Zhang Zhi jing, Cai Shao peng and Li Yang \\ School of Mechanical Engineering, Beijing Institute of Technology, Beijing, P.R. China
}

\begin{abstract}
Microassembly process is one of the difficulties in the field of manufacturing. According to the structural characteristics and assembly process difficultly of 3D meso-scale micro-devices and the requirement of multi-axis control in coaxial alignment assembly, this paper developed a vision and force feedback control method oriented to Microassembly System with Coaxial Alignment. This system combines multi-axis motion control with machine vision and force sensors, which can complete nondestructive assembly of parts and increase the precision of microassembly process into micron level.
\end{abstract}

\section{Introduction}

In the current microelectromechanical systems (MEMS) research field, high precision MEMS composed of mesoscale micro devices is the mainstream of civil and national defence micro manufacturing systems [1].

In the core equipment with urgent needs of the science and technology project and high precision MEMS manufacturing, guide instruments in aerospace and aviation field, intelligent fuze and NIF ignition targets, all belong to the meso-scale micro device demanding assembly gaps. The assembly level of those devices highly restricts the development of the high-end manufacturing of new generation into high precision and high stability. The assembly gaps of those instruments and components must be strictly restricted in micron level, and the alignment detection precision must be restricted in submicron level.

According to the features of those components and systems, an in-house microassembly system with coaxial alignment function (MSCA) was proposed [2]. This microassembly system uses a CCD camera to detect the position and pose of parts, and then assembles in the way of man-machine cooperation. Different target parts were assembled into the base part in the assembly process.

Microassembly process with coaxial alignment function is divided into two stages: the first stage, before target part contacting with base part, the visual feedback can detect the position and variation deviation between the two parts; the second stages, withdrawing the vision system and begin to assembly, during the target part contacting with base past, the force feedback can detect the position and variation deviation between the two parts further. In order to achieve non-destructive microassembly, this paper proposed vision and force feedback control method for coaxial alignment.
This paper is organized as follows. Section 2 reviews the motion platforms in the MSCA, vision system, and control system. The vision feedback and the force feedback are discussed in section 3. Section 4 describes the experiment process and result of this work. Section 5 concludes the work and gives the future direction of our work.

\section{Background}

\subsection{MSCA}

MSCA is used for the micro scale robotic assembly operation through the way of man-machine cooperation. As shown in Fig. 1.

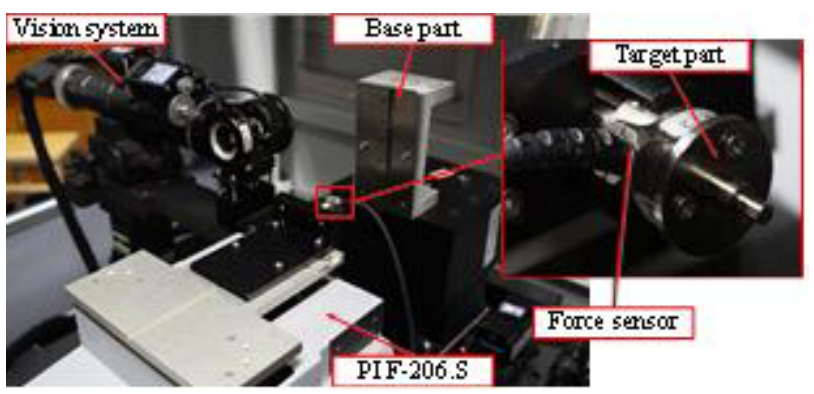

Figure 1. Microassembly system with coaxial alignment function.

\subsubsection{Composition of the manipulator}

This manipulator uses a combination of macro and micro movement to achieve the combination of a long working stroke and short precision feed to adjust the position and orientation of the part. Macro moving platforms, which supports two vacuum absorption micro-grippers and 6DOF force sensors (ATI NANO17, resolution: $0.3 \mathrm{mN}$, 
$0.004 \mathrm{~N} \cdot \mathrm{mm}$ ), uses THK precision module (positioning precision: $0.02 \mathrm{~mm}$, repeated positioning precision) and grating feedback in the axial feed, closed-loop configuration open loop control mode in order to record the precise macro feed amount. Micro moving platform uses a hexapod 6-DOF precision alignment worktable, PI F-206.S, which supports a mechanical fixture. The former module can provide $100 \mathrm{~mm}$ large motion range with a resolution of $0.02 \mathrm{~mm}$ while the latter worktable can only translate $\pm 5.7 \mathrm{~mm}$ motion range along the $\mathrm{x}-$, $\mathrm{y}-$, and $\mathrm{z}-$ axes with the translational resolution of $0.1 \mu \mathrm{m}$ and can also rotate $5.5^{\circ}$ along $\gamma-, \beta$-, and $\alpha$-axes with the rotational resolution of $2 \mu \mathrm{rad}$.

\subsubsection{Vision system}

A vision system is installed onto the MSCA to provide visual feedback for the assembly through the way of man-machine cooperation. The vision system consists of a cube prism, monochrome Basler scA1600-14gm digital camera (resolution: $1626 \times 1236$, optical size: 1/1.8', pixel size: $4.4 \mu \mathrm{m}$ ), auto zoom and focus lens remodeling by Navitar Zoom 12X. The working distance is 86 , optical magnification is $0.58 \mathrm{X} \sim 7 \mathrm{X}$. Two white point light sources, OPT-RI5090 and OPT-RI5045, is used as the light source.

\subsection{Control system}

MSCA has to control more than 20 axis movements, and how to guarantee the good stability and coordination between different axes has become one of the difficulties in current High Precision Micro Assembly Control System. There are five kinds of typical control system architecture: (1) base on CNC system; (2) base on PLC;

(3) base on general controller; (4) base on computer and special controller; (5) base on field bus [3]. Among them, the forth architecture is more excellent than others in multi-axis motion control, hardware compatibility, scalability and development platform. According to the features of MSCA, this paper developed an "industrial computer+ special controller+ UMAC" open control system based on the forth architecture.

\subsubsection{Hardware selection}

Advantech 610L is used as industrial control machine which has a large number of various interfaces, not only meets the current requirements, but also extends other functions through the rest interfaces according to followup research and development. We select Yaskawa SGD7S servo drives as the macro moving parts (highspeed, high frequency and high precision positioning control) to control. The F-206 Hex Align controller for micro moving parts (PI F-206.S) performs sophisticated six-space coordinate transformations and path planning internally, automatically. Multi-axis motion control card selects UMAC, which has good openness and scalability, can control up to 32 axes simultaneous movement.

\subsubsection{Software design}

In the "industrial computer+ special controller+ UMAC" architecture, industrial computer as the host, whose task is to the communicate with special controllers and data acquisition cards, get the system status in the assembly process, allocate control tasks and coordinate lower controllers simultaneously to complete the assembly task through the multi-tasking operating system. Special controllers belong to execution layer, receive host commands, and perform real-time control function according to specified parameters.

Depending on the different functions to be realized, control system application software includes modules shown in Fig. 2.

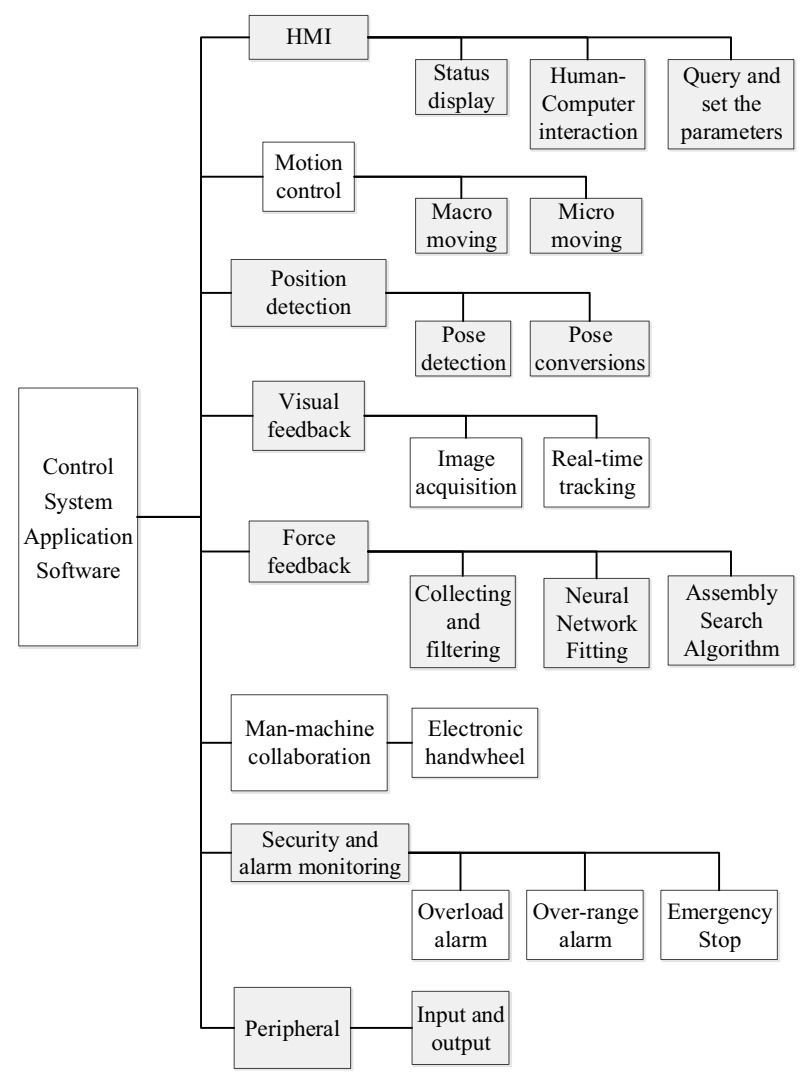

Figure 2. The architecture of control system application software.

\section{Vision and force feedback}

\subsection{Vision feedback}

Principle of coaxial optical alignment detection is shown in Fig. 3. A special prism was integrated to capture the images of the base part and target parts, which has two special surfaces: Surface 1 was a fully reflecting surface. Surface 2 was coated with a semi-anti-semi permeable. The CCD camera could capture the image of the target part and the base part simultaneously through the prism and obtain the base part and the target part's position corresponding relationship in the same image plane in different pose. As can be seen from the Fig. 3, optical path difference exists in the process of completing the image capture work of the target part and the base part. If we want to get a clear image of base part and target part 
simultaneously, wo must do the initial calibration before alignment to eliminate the optical path difference and obtain a clear image.

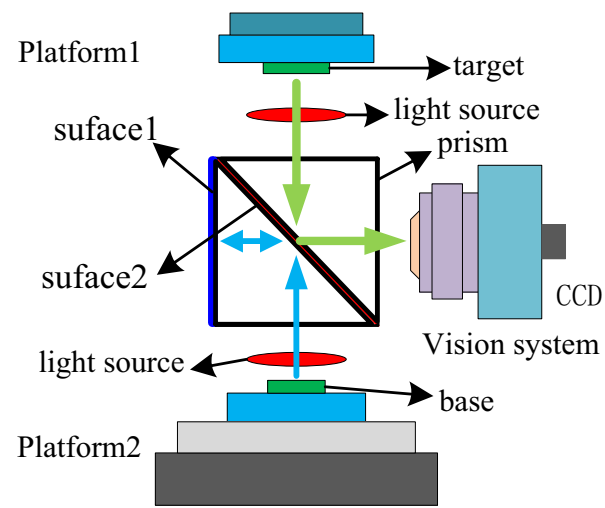

Figure 3. Principle of coaxial optical alignment detection.

We can see the optical path difference between the target part and the base part of the cube prism from Fig. 3, the light of target part reflected directly to the camera image plane through the surface 2 of the prism, the light of the base part reflected to the camera image plane through the surfacel in the left of the prism after penetrating the surface 2 . In that way, the light energy losses of the target part and the base part are different in cube prism. Therefore, the target part and the base part must be taken in the way of individually lighting, and the system uses time-sharing imaging control strategy. Coaxial alignment system is shown in Fig. 4.

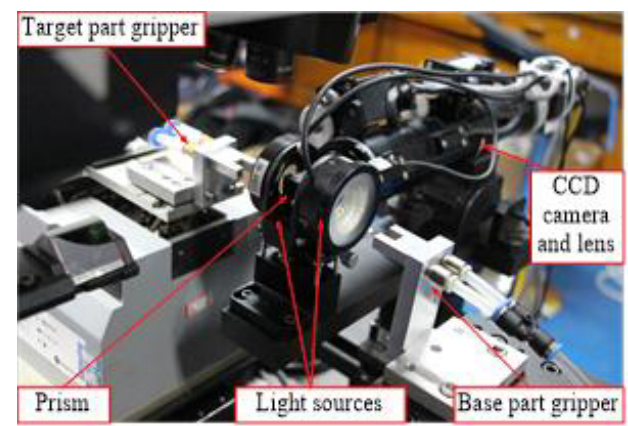

Figure 4. Coaxial alignment system.

Lens focusing and zooming controller use Navitar's Motor driver 1-62506, This driver application allows the user to configure and control a two motor (zoom and focus) system complete with limit sensors. The controller is connected via serial communication. Light source control selects the digital voltage controller OPTDP1024-4, its performance parameters are shown in Table 1.

Table 1. Lights source controller performance parameters.

\begin{tabular}{|c|c|}
\hline & Parameter \\
\hline Model & OPT-DP1024-4 \\
\hline Power supply & AC100 240(V) \\
\hline Voltage output & $14.0 \mathrm{~V} \sim 24.0(\mathrm{~V})$ \\
\hline Brightness level & 256 \\
\hline Number of Channels & 4 \\
\hline Single Output & $0.65(\mathrm{~A})$ \\
\hline
\end{tabular}

\subsection{Force feedback}

The main function of force feedback is threshold alarm during the parts contacting. As shown in figure 5, in the process, adjusting the assembly micro devices space pose, calculating the stress of the micro devices of various pose, finding the maximum stress, comparing with the permissible stresses of materials, obtaining the range of stresses that realize nondestructive assembly and calculating the corresponding range of assembly force. According the calculation results, microassembly with coaxial alignment system can realize micro devices nondestructive assembly on the premise of the assembly force in the safety range. In the assembly process, strictly monitoring the force of the specific direction whether exceeds the safety threshold or not.

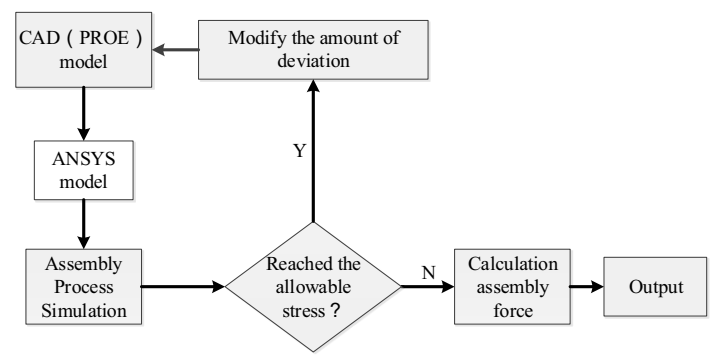

Figure 5. Step of virtual detection.

\section{Experimental results and discussion}

Experiment based on MSCA, the author conducted coaxial alignment assembly experiment. In order to verify the assembly accuracy of MSCA, we specially designed and processed $3 \mathrm{~mm}$ diameter shaft-hole array assembly parts of high-precision assembly, as shown in Fig. 6. We selected the 3rd shaft and the 3rd hole as a precision verification target, which single coordinate clearance is less than $2 \mu \mathrm{m}$. The $3 \mathrm{rd}$ hole' $\mathrm{s}$ diameter is $2.9994 \mathrm{~mm}$, cylindricity is $0.0021 \mathrm{~mm}$; the $3 \mathrm{rd} \mathrm{shaft}^{\prime} \mathrm{s}$ diameter is $2.9971 \mathrm{~mm}$, cylindricity is $0.0012 \mathrm{~mm}$.
(a)Hole array

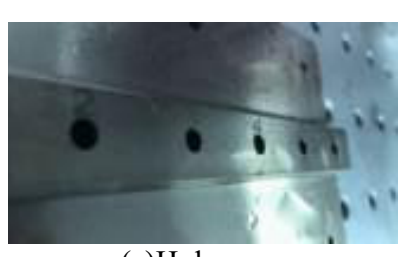

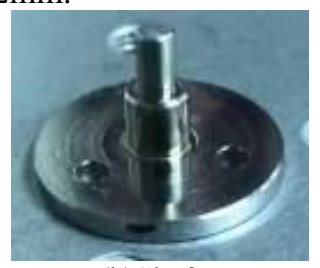

(b)Shaft
Figure 6. $3 \mathrm{~mm}$ diameter shaft-hole array assembly parts.

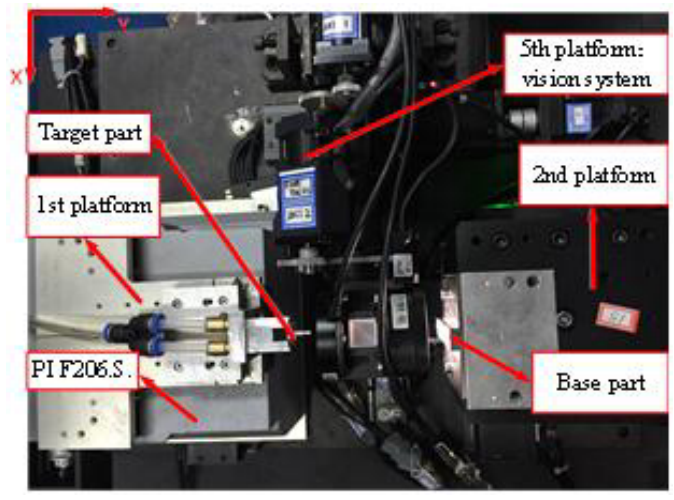

Figure 7. Assembly experimental devices. 
Coaxial alignment assembly experimental devices are shown in Fig. 7. Using vacuum adsorption method to grip the parts, the target part is fixed to the 1st platform; the base part is fixed to the 2nd platform. Vision system is in the 5th platform, which perpendiculars to the 1st and 2nd platforms' console axis in a horizontal plane. PI F-206.S is placed on the 1st platform to adjust the target part's 6DOF pose in micro-scale.

Coaxial alignment assembly experimental flow is shown in Fig. 8.

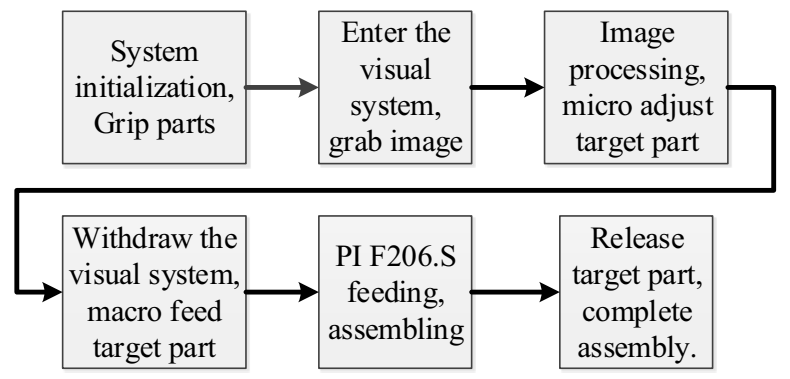

Figure 8. Coaxial alignment assembly experimental process

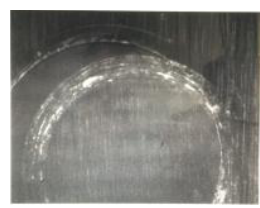

(a)Before alignment

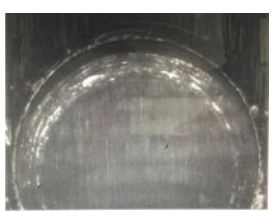

(b) Aligning

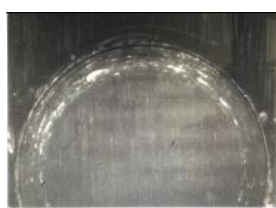

(c) Alignment completed
Figure 9. Images change process in coaxial alignment experiment.

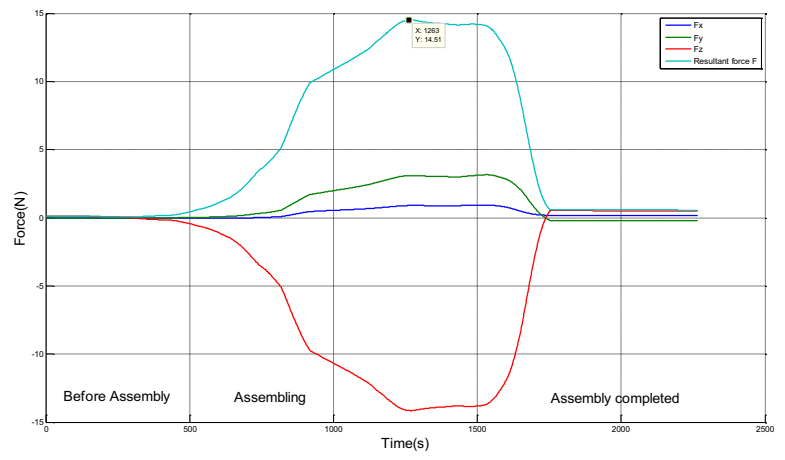

Figure 10. The changing process of the amount of the forces.

During the experiment, CCD camera captured the images of the shaft and the hole, as shown in Fig. 9. The image of the shaft and hole which is before aligned are shown in Fig. 9. (a), apparently there is deviation between the parts. Fig. 9. (b) shows the image of the shaft and hole in the process that PI F206.S is adjusting the target part's pose according to visual feedback. Fig. 9. (c) is the state of the shaft hole after coaxially aligning, which means that the target part and the base part have completed the coaxial alignment. During assembly, the changing process of the amount of the forces is shown in Fig. 10, it can be seen from the Fig. 10 that the maximum resultant force is $14.51 \mathrm{~N}$, which is far less than the allowable assembly force. Experiments show that, MSCA can complete nondestructive assembly of parts in the micron level.

\section{Conclusion}

Oriented to MSCA, the control system is capable of controlling multiple axes of motion simultaneously which was developed using "industrial computer+ special controller+ UMAC" open control system architecture. Combining with different detection equipment such as machine vision and force sensor, the system can complete nondestructive assembly of parts and increase the precision of microassembly process into micron level, through the way of man-machine cooperation. In the future work, several researches need to be done on visual servoing and finding out the relationship between the micro-force and micro-displacement. We have to do some improvements to increase the automation of the MSCA system.

\section{Acknowledgements}

This work was financially supported by the National Natural Science Foundation of China (51575052) and (51405025). Any opinions, findings, and conclusions or recommendations expressed in this material are those of the authors and do not necessarily reflect the views of the National Natural Science Foundation of China.

\section{References}

1. Ehmann.Kornel, DeVor.Richard, Kapoor.Shiv, Ni. Jun: Workshop: Micro/Meso-Mechanical Manufacturing, Evanston, llinois, USA, Northwestern University, (2000).

2. Xin Ye, Jun Gao, Zhi Jing Zhang, Applied Mechanics and Materials, 487, 678-681(2014)

3. Spear.Mike, Process Engineering, 75, 38-39 (1994). 